\title{
Exploring potential jet modification in small collision systems with two particle correlations at PHENIX
}

\author{
Cheuk-Ping Wong ${ }^{a, b, 1, *}$ \\ ${ }^{a}$ Los Alamos National Laboratory, \\ Los Alamos, NM, USA \\ ${ }^{b}$ Georgia State University, \\ Atlanta, GA, USA \\ E-mail: cpwong@lanl.gov
}

Flow results in the small collision systems at RHIC and the LHC indicate QGP droplets are formed in these small systems. Measurements of jet $R_{p A}$ on the other hand shows that there is no modification to the jets. However, non-zero $v_{n}$ values for high momentum particles, which in $A+A$ collisions are attributed to path-length dependent energy loss, have been observed in small systems. To further probe possible modification to jet particles, PHENIX measures the relative modification of the near and away-side jet particles in $\pi^{0}$-hadron correlations in $200 \mathrm{GeV} d+A u$ and ${ }^{3} \mathrm{He}+\mathrm{Au}$ collisions. Similar modification as observed in $A+A$ collision, such as suppression of high $p_{T}$ particle yield and enhancement of low momentum associated with the high $p_{T}$ trigger have been observed in $d+A u$ collisions. These surprising results have now also been seen in ${ }^{3} \mathrm{He}+\mathrm{Au}$ data. These PHENIX measurements and possible interpretations of these intriguing results are presented.

HardProbes 2020

1-6 June 2020

Austin, Texas

${ }^{1}$ For the PHENIX Collaboration

* Speaker 


\section{Introduction}

With the versatility in both energy range and collision species, RHIC delivers a wide range of collisions in different system size. Conventionally, the small collision systems are used to study the cold nuclear matter effects which are important to extract the hot nuclear matter effect coming from the production of quark-gluon plasma (QGP) in the large systems. However, the recent discovery of the QGP droplet reported by the PHENIX collaboration [1] changed the perspective in the small system studies. The results in [1] showed the second, third and fourth order flow harmonic coefficients in $p+A u, d+A u$ and ${ }^{3} \mathrm{He}+\mathrm{Au}$ measurements are non-zero. Moreover, the flow harmonic coefficients show system size dependence. While the effects on partonic energy loss of the QGP droplet in the small system measurements are unclear, more experimental results can help theorists to constrain their energy loss models in QGP studies. This presentation summarized the recent jet modification studies in small systems, including the $p+A, d+A u$ and ${ }^{3} \mathrm{He}+\mathrm{Au}$ collisions at $200 \mathrm{GeV}$ data collected by PHENIX, using $\pi^{0}$-hadron correlations. Discussions of system size dependence was also covered with the comparisons of different collisions species.

\section{Yield Modifications in small systems}

To study the yield modification in the $A+A$ collisions with respect to the $p+p$ collisions, $I_{A A}$ was measured in two-particle correlations analysis. $I_{A A}$, which is equivalent to the modifications of fragmentation function, is defined as the ratio of integrated yield, $Y_{A A}$, of the correlation functions in $A+A$ to the integrated yield, $Y_{p p}$, in $p+p$, that is $I_{A A}=Y_{A A} / Y_{p p}$. The recent away-side $I_{A A}$
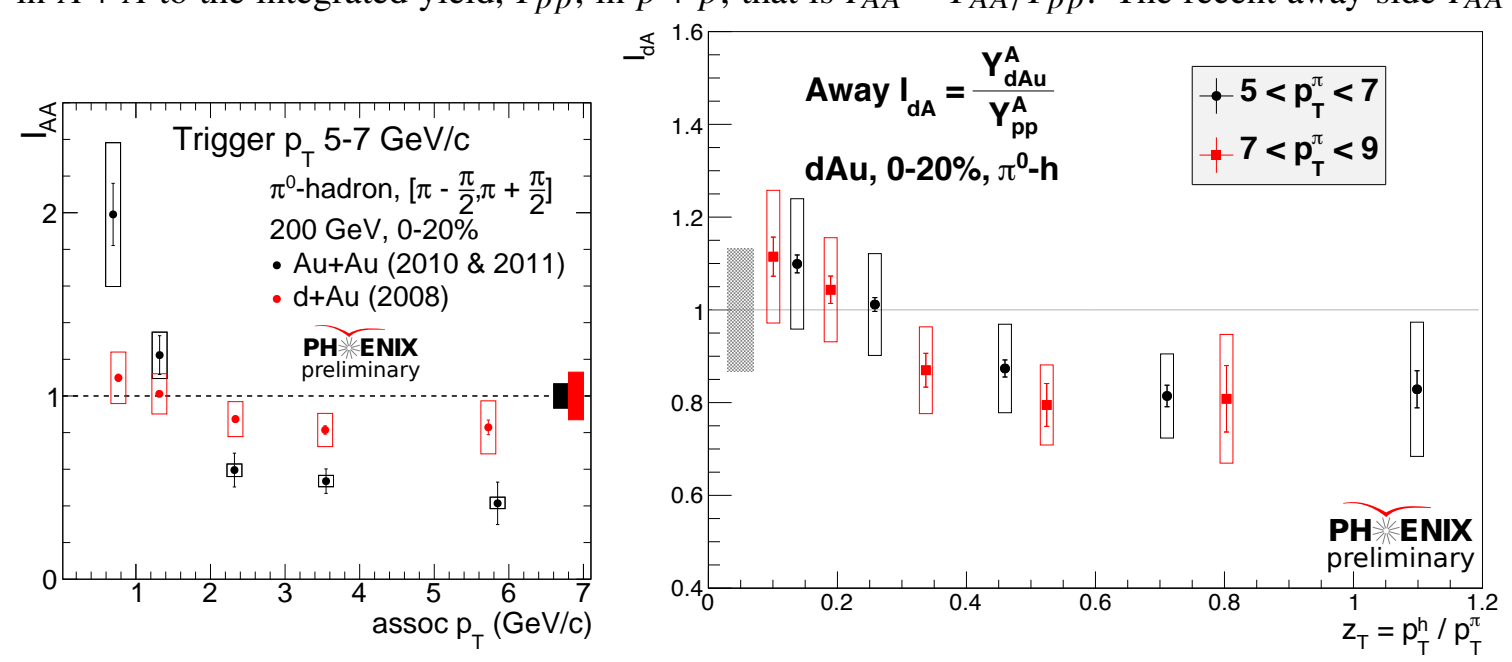

Figure 1: Left: comparison of away-side $I_{A A}$ between $A u+A u$ and $d+A u$ collisions at $200 \mathrm{GeV}$ [2]. Right: away-side $I_{A A}$ as a function of $z_{T}$ in $d+A u$ collisions at $200 \mathrm{GeV}$ [3]. Statistical, systematic and global scale uncertainties are drawn as vertical bars, open boxes and filled boxes, respectively.

results of $\pi^{0}$-hadron correlations in $A u+A u$ collisions at $200 \mathrm{GeV}$, obtained from the data collected in RHIC Year-2010 and 2011 runs, clearly show yield modification of particles from jets. The yield of high $p_{T}$ particles was found to be suppressed, while the one of low $p_{T}$ particles was enhanced, which is consistent with the jet quenching scenario; the partons lose energy when they traverse the QGP. As shown on the left of Figure 1, the away-side $I_{A A}$ from $d+A u$ collisions collected in 
2008 is closer to unity than the $A u+A u$ results across the associate $p_{T}$ range indicating that jet quenching effect is stronger in the $A u+A u$ collisions than in the $d+A u$ collisions.

Although the away-side $I_{A A}$ in $d+A u$ collisions hints to yield suppression at high $p_{T}$ as shown in Figure 1, the $I_{A A}$ also agree with unity due to sizeable systematic and global scale uncertainties. To cancel some of the systematic uncertainties, a double ratio, $R_{I}$, is introduced. The double ratio, $R_{I}=I_{A A}^{A w a y} / I_{A A}^{N e a r}$, is the ratio of $I_{A A}$ on the away-side to the $I_{A A}$ on the near-side. Since the near-side $I_{A A}$ is consistent with unity in small systems, $R_{I}$ shown in Figure 2 is consistent with $I_{A A}$ shown on the right of Figure 1. Furthermore, systematic uncertainties are reduced in $R_{I}$ compared to $I_{A A}$, as the near and away-side $I_{A A}$ are obtained from the same measurement. Figure 2 shows that the $R_{I}$ is clearly above 1 at low $z_{T}$ and below 1 at high $z_{T}$ indicating the enhancement of soft particles and the suppression of high $p_{T}$ particles.

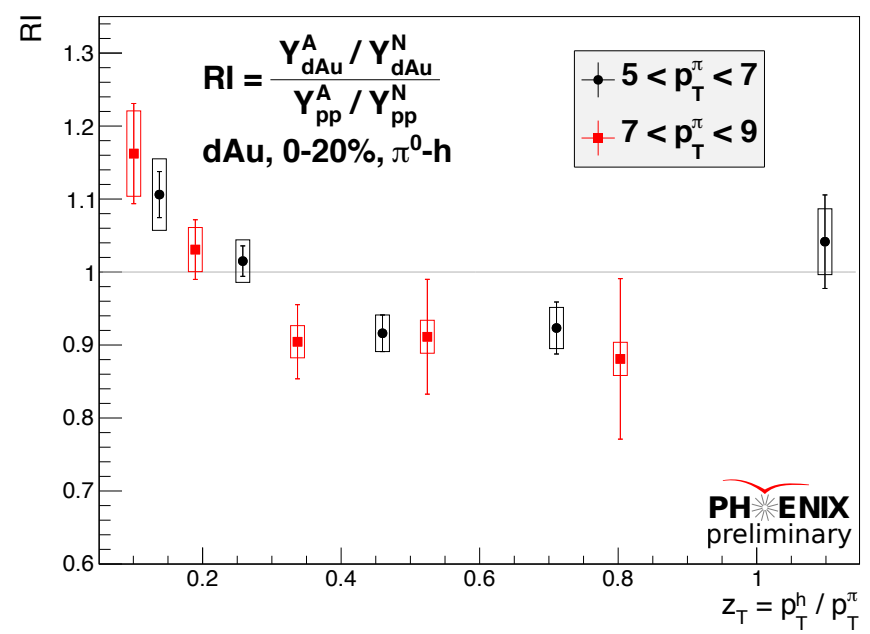

Figure 2: $R_{I}$ as a function of $z_{T}$ in $d+A u$ collisions at $200 \mathrm{GeV}$ [3]

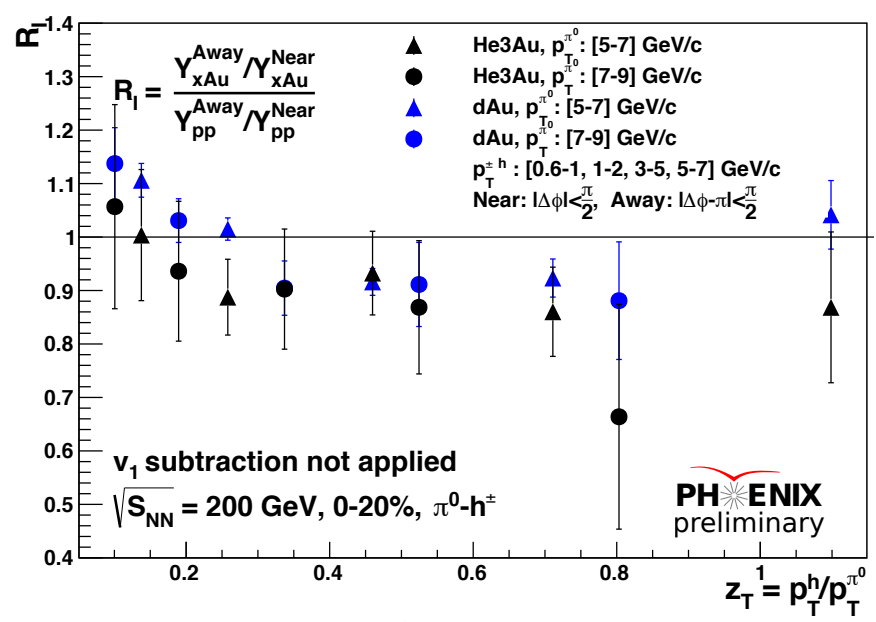

Figure 3: Comparison of $R_{I}$ between $d+A u$ and ${ }^{3} \mathrm{He}+A u$ collisions at $200 \mathrm{GeV}$. Systematic uncertainties are not shown [3].

To study system size dependence, $R_{I}$ from ${ }^{3} \mathrm{He}+\mathrm{Au}$ collisions at $200 \mathrm{GeV}$ is also measured to compare with the $d+A u$ results as shown in Figure 3. Although the results from $d+A u$ are consistent with the ${ }^{3} \mathrm{He}+\mathrm{Au}$ results considering the size of the uncertainties, the $\mathrm{R}_{I}$ from ${ }^{3} \mathrm{He}+\mathrm{Au}$ is systematically smaller than the $d+A u$ results. This difference is more clear as shown in the ratio 
plot in Figure 4. The $R_{I}$ ratio of ${ }^{3} \mathrm{He}+\mathrm{Au}$ to $d+A u$ at high $z_{T}$ estimated by a straight line fit is $0.876 \pm 0.061$, which hints to the stronger suppression of hard jet particles in ${ }^{3} \mathrm{He}+\mathrm{Au}$ collisions.

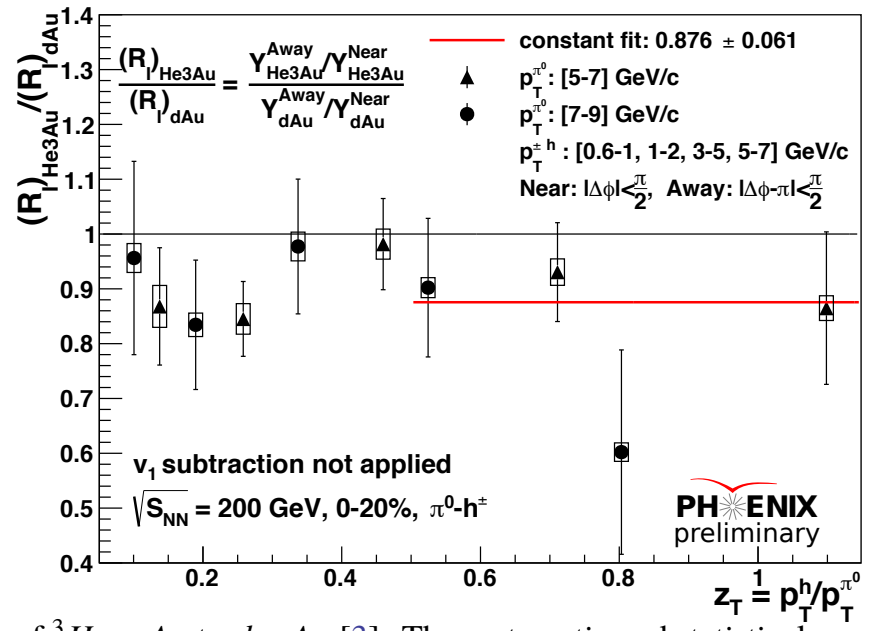

Figure 4: Ratio of $R_{I}$ of ${ }^{3} \mathrm{He}+\mathrm{Au}$ to $d+A u$ [3]. The systematic and statistical uncertainties from each RI are added in quadrature individually.

\section{Momentum Broadening in Small Systems}

Recent results of $I_{A A}(\Delta \phi)$ from Au+Au collisions at $200 \mathrm{GeV}$, as shown in Figure 5, demonstrated that the quantities are useful to look for the location of enhancement or depletion of the yield within a jet. These new results shows enhancement of soft jet particles at the skirt of the jet, indicating angular broadening in $A u+A u$ collisions.

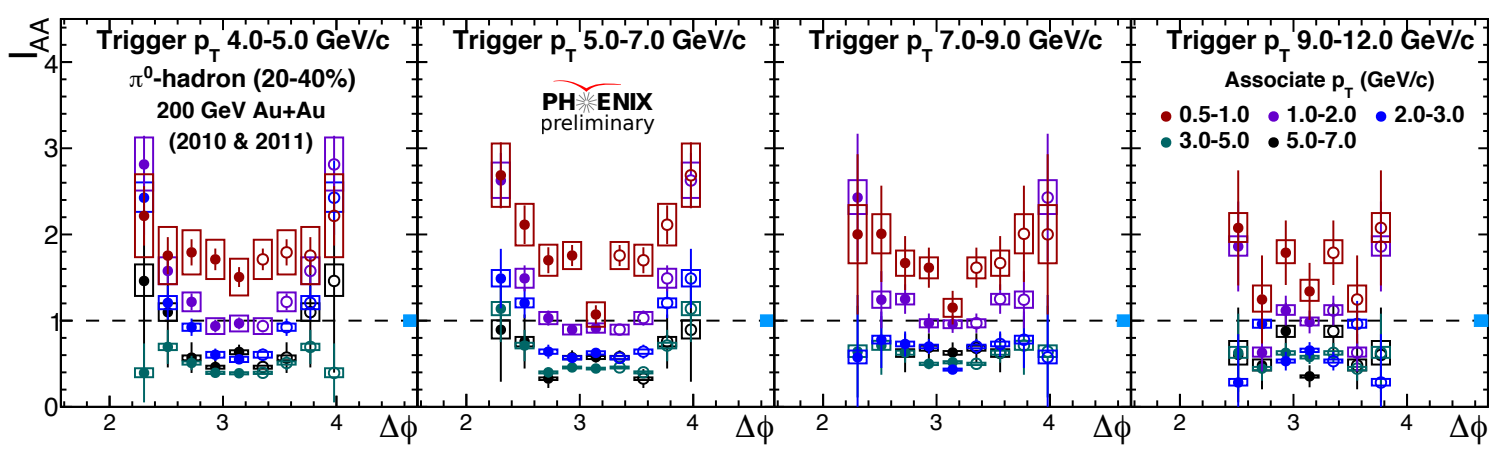

Figure 5: $I_{A A}$ as a function of $\Delta \phi$ measured in $A u+A u$ collisions at $200 \mathrm{GeV}$.

Jet broadening can also be studied in momentum space using $p_{\text {out }}$ observable. $p_{\text {out }}=$ $p_{T}^{a s s o c} \cdot \sin \Delta \phi$ is the transverse component of the associate particle transverse momentum, $p_{T}^{\text {assoc }}$, with respect to the trigger particle transverse momentum, $p_{T}^{\text {trig }}$, which provides a handle on the jet axis in the two-particle correlation analysis. The difference of squared Gaussian width of $p_{\text {out }}$ distributions between $p+A$ and $p+p$ measurements as a function of number of collisions, $N_{\text {coll }}$, is shown in Figure 6 [4]. $x_{E}$ in Figure 6, which is defined as $x_{E}=-\left(\left|p_{T}^{\text {assoc }}\right| /\left|p_{T}^{\text {trig }}\right|\right) \cos \Delta \phi$, is the longitudinal fraction of the associate particle $p_{T}$ to the trigger particle $p_{T}$. Therefore, $x_{E}$ resembles the $\mathrm{z}$ parameter in the fragmentation functions. Figure 6 shows that the width of the $p_{\text {out }}$ distribution is broader in $p+A$ than in $p+p$ measurement indicating momentum broadening. Furthermore, the differences of the width increases with a higher $N_{\text {coll }}$ indicating system size dependence. The 
possible explanations of the system size dependence include intrinsic $k_{T}$ effect, or potential energy loss. The influence of the underlying flow is negligible as shown in the analysis.

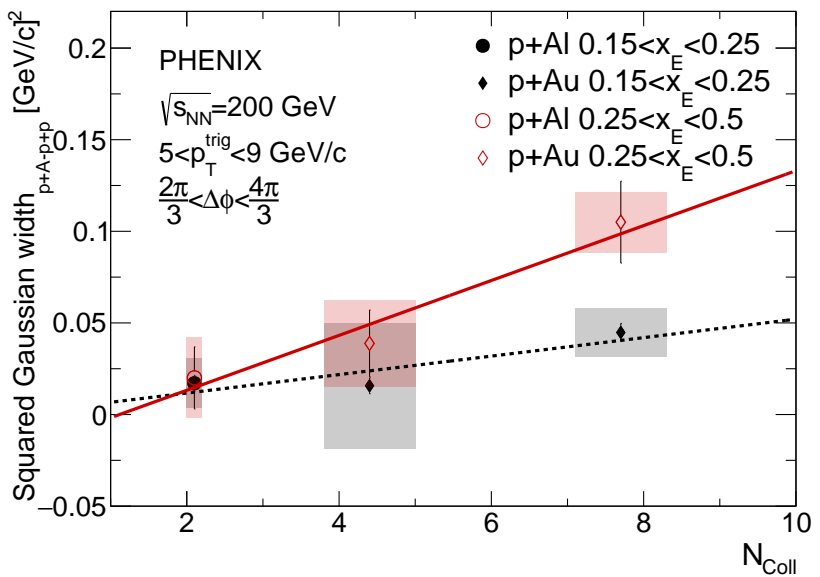

Figure 6: Difference of squared Gaussian width of $p_{\text {out }}$ distributions between $p+A$ and $p+p$ measurements as a function of number of collisions, $N_{\text {coll }}[4]$.

\section{Summary}

This presentation summarized recent yield and momentum modifications of jets in small system collisions at $200 \mathrm{GeV}$ using $\pi^{0}$-hadron correlations in PHENIX data. The double ratio $R_{I}$ is introduced to study away-side yield modifications in small systems. $R_{I}$ results from both $d+A u$ and ${ }^{3} \mathrm{He}+\mathrm{Au}$ collisions show away-side high $p_{T}$ particles are suppressed. The $p_{\text {out }}$ results from $p+A$ collisions indicating that there is additional momentum broadening compared to $p+p$ results.

More interestingly, like the flow measurements in small systems, both yield and momentum modifications show system size dependence. The momentum broadening increases with number of collisions in $p+A$ collisions, while the $R_{I}$ results from ${ }^{3} \mathrm{He}+\mathrm{Au}$ collisions are systematically lower than $d+A u$ collisions, indicating stronger hard jet particles suppression in ${ }^{3} \mathrm{He}+\mathrm{Au}$ collisions. While these results hint at possible jet modification in small systems, more studies and model comparisons are valuable to clarify the nuclear matter effects in small systems.

\section{Acknowledgments}

This author's work was supported by the National Science Foundation [grant number 1848162].

\section{References}

[1] C. Aidala et al., Creation of quark-gluon plasma droplets with three distinct geometries, Nat. Phys. 15 (2019) .

[2] C. Wong, Phenix results of $\pi^{0}$-hadron correlation, PoS High-pT2019 (2020) 4.

[3] A. Pun, Gamma-hadron and related Two-Particle Azimuthal correlations studies in PHENIX, PoS High-pT2019 (2020) 1.

[4] C. Aidala et al., Nonperturbative-transverse-momentum broadening in dihadron angular correlations in $\sqrt{s_{N N}}=200 \mathrm{GeV}$ proton-nucleus collisions, Phys. Rev. C 99 (2019) 044912. 